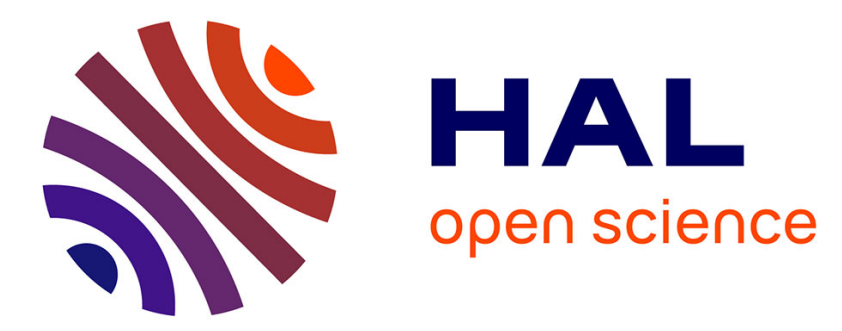

\title{
Dispositif expérimental destiné à l'étude des collisions atomiques et moléculaires aux énergies thermiques et suprathermiques
}

\author{
J. Cuvellier, J.M. Mestdagh, J. Berlande, P. de Pujo, A. Binet
}

\section{To cite this version:}

J. Cuvellier, J.M. Mestdagh, J. Berlande, P. de Pujo, A. Binet. Dispositif expérimental destiné à l'étude des collisions atomiques et moléculaires aux énergies thermiques et suprathermiques. Revue de Physique Appliquée, 1981, 16 (12), pp.679-686. 10.1051/rphysap:019810016012067900 . jpa00244962

\section{HAL Id: jpa-00244962 https://hal.science/jpa-00244962}

Submitted on 1 Jan 1981

HAL is a multi-disciplinary open access archive for the deposit and dissemination of scientific research documents, whether they are published or not. The documents may come from teaching and research institutions in France or abroad, or from public or private research centers.
L'archive ouverte pluridisciplinaire HAL, est destinée au dépôt et à la diffusion de documents scientifiques de niveau recherche, publiés ou non, émanant des établissements d'enseignement et de recherche français ou étrangers, des laboratoires publics ou privés. 


\title{
Dispositif expérimental destiné à l'étude des collisions atomiques et moléculaires aux énergies thermiques et suprathermiques
}

\author{
J. Cuvellier, J. M. Mestdagh, J. Berlande, P. de Pujo et A. Binet \\ Centre d'Etudes Nucléaires de Saclay, Service de Physique des Atomes et des Surfaces, 91191 Gif-sur-Yvette Cedex, France
}

(Reçu le 26 juin 1981, accepté le 18 septembre 1981)

\begin{abstract}
Résumé. - Le dispositif expérimental qui est décrit dans cet article est destiné à l'étude des collisions atomiques et moléculaires aux énergies thermiques et suprathermiques. Utilisant deux générateurs de faisceaux supersoniques de particules et un laser pour l'excitation lumineuse, il permet d'étudier quantitativement les processus collisionnels se produisant entre une particule excitée et une autre particule, lorsque l'énergie relative des deux partenaires varie de quelques centièmes d'électron-volt à quelques électron-volts. Il a été conçu et réalisé de telle sorte qu'il soit possible de préparer les particules dans des états quantiques et dans des conditions d'énergies cinétiques bien définis. Outre la description détaillée du dispositif expérimental, nous indiquons les techniques de mesures qui ont été utilisées. Pour illustrer les possibilités de ce dispositif quelques résultats expérimentaux originaux relatifs aux transferts de structure fine alcalin-gaz rare et alcalin-molécule sont indiqués.
\end{abstract}

\begin{abstract}
The experimental apparatus described in this paper is devoted to the study of atomic and molecular collisions at thermal and suprathermal energies. Using two supersonic particle beam generators and a laser for beam excitation, this apparatus allows a quantitative measurement of the processes involved in collisions between an electronically excited particle and a target atom or molecule. The relative energy of the collision partners may be varied between $10^{-2} \mathrm{eV}$ to several $\mathrm{eV}$. This apparatus was designed to prepare particles in well defined translational energy. In addition to the detailed description of the apparatus, the measurement technics are also designed. As exemple of the types of studies possible with this apparatus, some recent experimental results concerning the fine structure transition process in alkali, resulting from collision with rare gases and molecules, are given.
\end{abstract}

1. Introduction. - Notre laboratoire s'intéresse depuis une douzaine d'années aux problèmes posés par les collisions atome excité-atome ou atome excitémolécule à basse énergie [1]. Nos premiers résultats ont été obtenus en utilisant des expériences réalisées avec des cellules. Cette technique simple, mais néanmoins très sensible présente toutefois le double inconvénient de ne permettre qu'une exploration en énergie très réduite, imposée par les variations possibles de la température de la cellule, et de fournir des valeurs de sections efficaces moyennées sur toute la distribution des vitesses relatives des deux partenaires de la collision. Les importants développements technologiques de ces dernières années qu'il s'agisse de l'obtention des faisceaux lumineux intenses et monochromatiques (lasers à colorant) ou de la production de faisceaux monocinétiques de particules à haute densité (faisceaux supersoniques), nous ont permis d'envisager la réalisation d'un dispositif expérimental où les paramètres - vitesse et direction des particules, état quantique de l'atome excitéavant la collision sont parfaitement connus et où la gamme d'énergie explorable se trouve considérablement élargie. Par exemple, elle peut varier de quelques centièmes à quelques électron-volts lorsque l'on utilise la technique de l'ensemencement des faisceaux. La réunion sur un même dispositif des moyens précédemment énoncés, permet des mesures très précises des phénomènes collisionnels se produisant aux énergies thermiques et suprathermiques. C'est ainsi que la sensibilité et la linéarité d'un tel dispositif nous a permis d'effectuer des mesures quantitatives de sections efficaces s'étendant de quelques centièmes à plusieurs dizaines d'angstroms carrés. Ces mesures quantitatives permettent l'étude du comportement de nombreux couples atomes excitésatomes (ou molécules) en fonction de l'énergie de la collision et de comparer ces différents comportements entre eux. On peut ainsi comparer les résultats expérimentaux aux prédictions théoriques. Dans le cas où elles n'existent pas, les résultats expérimentaux peuvent contribuer à leur élaboration.

Avec ce dispositif il est possible de réaliser des expériences très diverses, nous l'avons pour notre 
part utilisé pour l'étude des transferts de structure fine induits dans un atome alcalin, lors d'une collision avec un atome ou une molécule. La méthode utilisée étant celle de la fluorescence sensibilisée [2, 3].

Les techniques de mesures et les éléments de ce dispositif expérimental qui sont devenus d'une utilisation courante dans les laboratoires ne seront décrits que sommairement, par contre nous insisterons davantage sur ceux qui conservent un caractère d'originalité.

2. Description du dispositif. - Il va donc s'agir de produire dans un volume d'interaction donné $\left(10 \mathrm{~mm}^{3}\right)$ la collision entre un atome alcalin excité et une autre particule dans l'état fondamental de telle sorte que les paramètres avant la collision soient parfaitement déterminés et d'analyser avec la plus grande précision les produits de la collision.

Le dispositif expérimental est schématisé sur la figure 1 , il se compose :

- d'un générateur de faisceau alcalin,

- d'un générateur à haute enthalpie avec son dispositif de mesure (temps de vol et densité des particules),

- d'une chambre de collision,

- d'un système d'excitation lumineuse et de collection de la lumière de fluorescence.

2.1 GéNérateur De JET alCalin. - Un des éléments principaux du générateur (Fig. 2) est le four. Nous nous sommes fortement inspiré de celui réalisé par H. O. Hoppe [4], mais comme nous désirions augmenter l'autonomie de son fonctionnement, nous avons augmenté la capacité du réservoir $\left(50 \mathrm{~cm}^{3}\right)$. La tuyère qui est du type convergente a un trou d'éjection de $240 \mu \mathrm{m}$. Pour éviter le bouchage de celui-ci il est nécessaire de surchauffer la tuyère de $100^{\circ} \mathrm{C}$ par rapport à la température du réservoir. D'autre part, l'emploi d'un alcalin très pur est recommandé. Celui que nous utilisons a un taux d'impuretés, hors alcalin, inférieur à $3 \times 10^{-4}$. Le tableau I donne les caractéristiques d'un faisceau de potassium dans le cas où l'excitation de l'alcalin est réalisé par un laser pulsé. L'utilisation d'un laser continu permet une forte réduction de la densité du faisceau ce qui entraîne une forte diminution de la consommation (de $10^{2}$ à $10^{3}$ ). Le rayonnement du four est limité par des écrans thermiques et par une chemise refroidie à l'eau. Toutes les pièces en contact avec le faisceau sont refroidies par une tresse en cuivre reliée à un cryostat rempli d'azote liquide.

Les caractéristiques indiquées dans le tableau I ont été mesurées avec un sélecteur de vitesse identique à celui utilisé par $\mathrm{H}$. O. Hoppe [4] et par un détecteur de Langmuir-Taylor pouvant se déplacer

Tableau I. - Caractéristiques du générateur de faisceau alcalin.

$\begin{array}{ll}\text { Alcalin } & \text { Potassium } \\ \text { Température du four } & 5000^{\circ} \mathrm{C} \\ \text { Pression de l'alcalin dans le four } & 30 \text { torr } \\ \text { Concentration à } 760 \mathrm{~mm} \text { de la tuyère } & 10^{9} \text { atomes } / \mathrm{cm}^{3} \\ \text { Vitesse hydrodynamique } & 930 \mathrm{~m} / \mathrm{s} \\ \begin{array}{l}\text { Dispersion des vitesses } \\ \text { totale à mi-hauteur) }\end{array} & \\ \begin{array}{l}\text { Consommation du four } \\ \text { Nombre de Mach }\end{array} & 26 \% \\ & 1 \mathrm{~g} / \mathrm{h} \\ \end{array}$

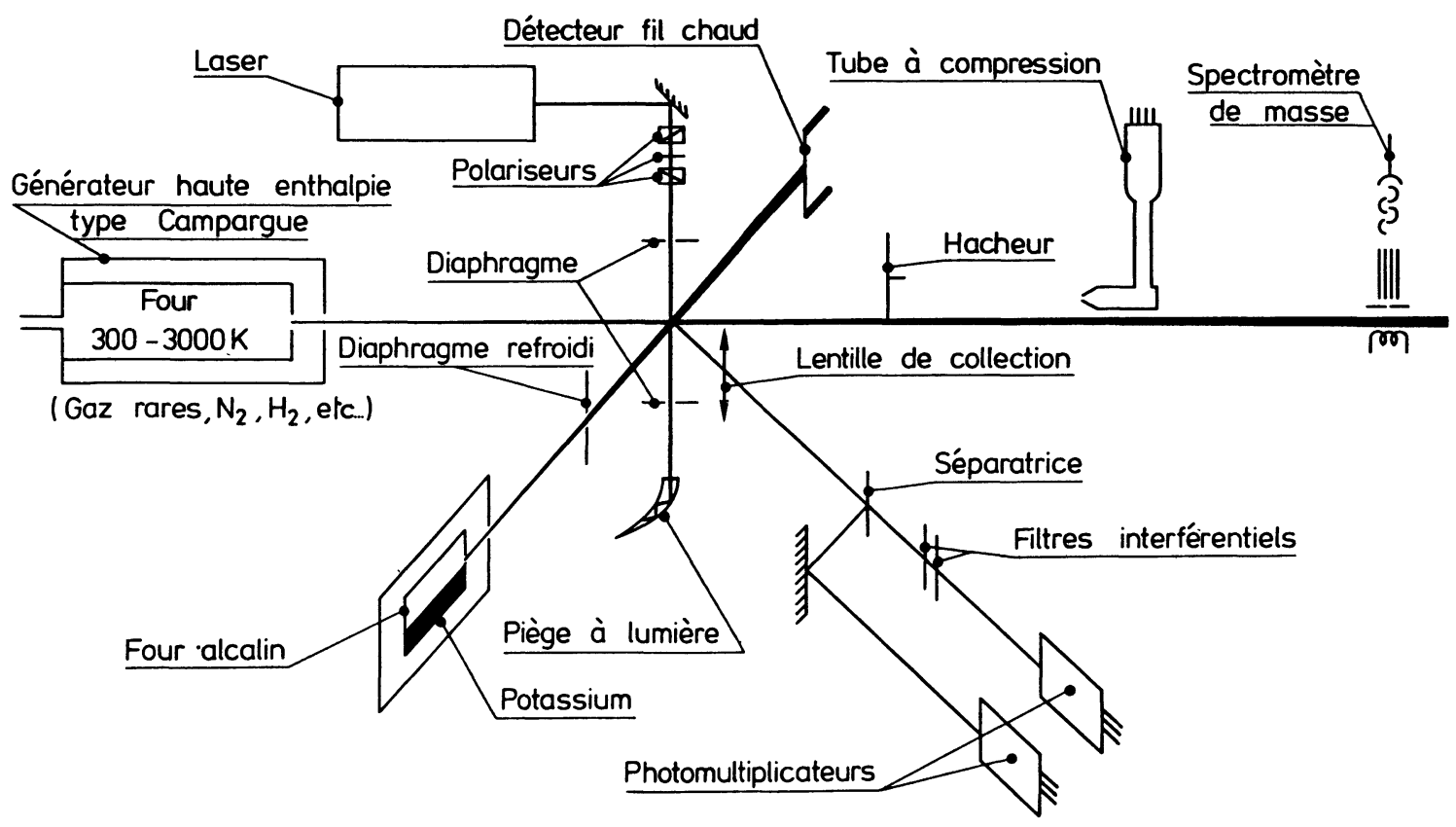

Fig. 1. - Schéma de principe de l'expérience.

[Schematic drawing of the experiment.] 


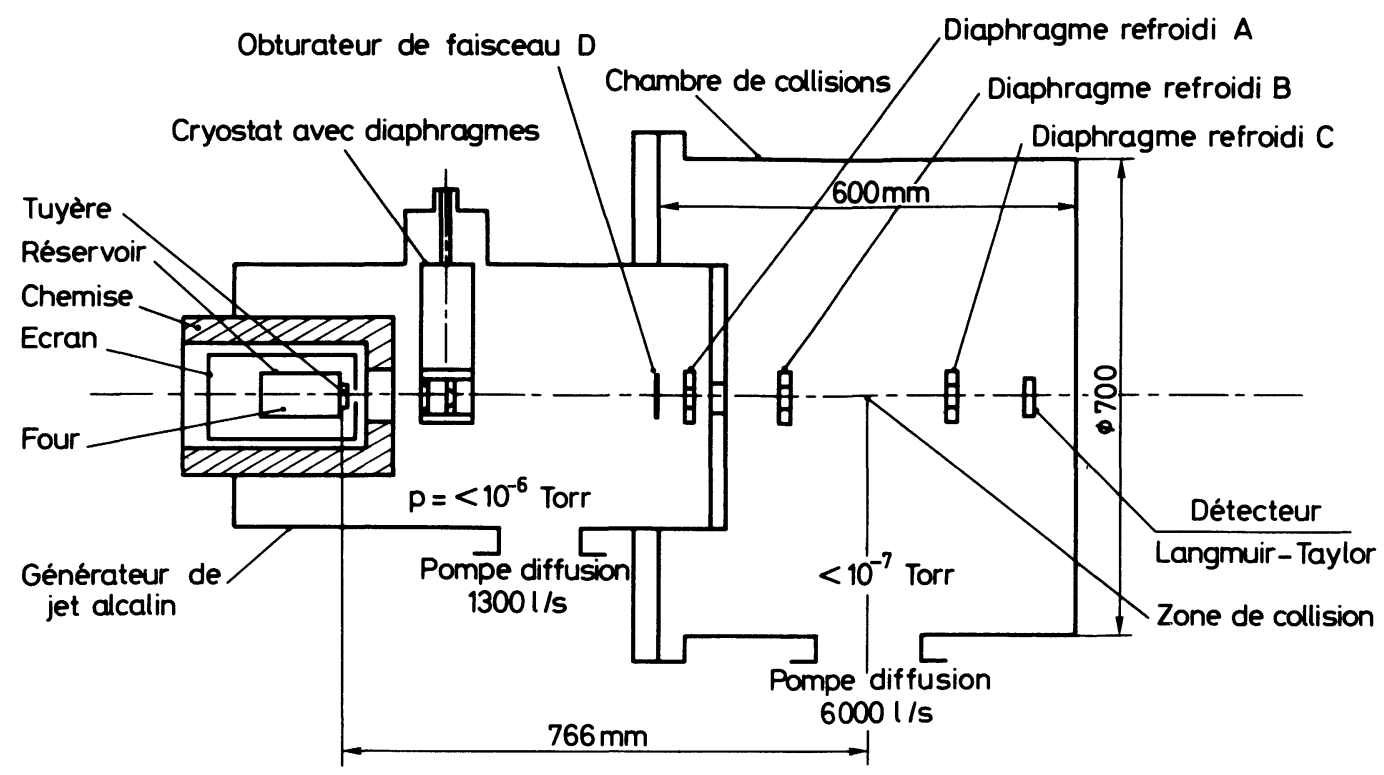

Fig. 2. - Générateur de faisceau alcalin et chambre de collisions.

[Alkali beam generator and collision chamber.]

dans un plan perpendiculaire au faisceau. Le filament de ce détecteur est constitué d'un mince ruban d'iridium de $5 / 100$ de millimètre d'épaisseur.

Un dispositif de pompage comprenant une pompe à diffusion munie d'un piège à azote liquide permet d'obtenir une pression meilleure que $10^{-6}$ torr.

\subsection{GÉNÉRateur a haute enthalpie TYPE CAM-} $P A R G U E$. - Il a été construit par le laboratoire de jets moléculaires du département de Physico-Chimie du C.E.N. Saclay dirigé par le Dr Campargue.

Ce générateur à haute enthalpie est destiné à produire un faisceau supersonique de gaz rares, de gaz moléculaires purs $\left(\mathrm{N}_{2}, \mathrm{H}_{2}, \mathrm{CO}, \mathrm{CO}_{2} \ldots\right)$ ou de mélanges de ceux-ci. Il se compose d'un four, d'un écorceur et d'un collimateur. La température du four pouvant varier de 300 à $3000 \mathrm{~K}$ il nous a été possible pour les différents gaz utilisés de faire varier l'énergie translationnelle des particules éjectées dans les gammes indiquées ci-dessous.

Hélium de 0,060 à $0,43 \mathrm{eV}$
Argon de 0,063 à $0,43 \mathrm{eV}$
Azote de 0,082 à $0,55 \mathrm{eV}$
Hydrogène de 0,066 à $0,45 \mathrm{eV}$

Pour un gaz moléculaire, la température rotationnelle peut varier dans des proportions importantes suivant les conditions de détente du gaz dans la tuyère. C'est ainsi que dans le cas de l'hydrogène, la température rotationnelle varie d'un ordre de grandeur $(100$ à $1000 \mathrm{~K})$ lorsque l'énergie translationnelle des particules éjectées varie de 0,06 à $0,45 \mathrm{eV}$. En ce qui concerne la température vibrationnelle on sait que cette température est pratiquement celle du four.

La technique de l'ensemencement d'un gaz lourd par un gaz léger permet d'augmenter très largement l'étendue de la gamme d'énergie explorable, c'est ainsi qu'en ensemençant un jet d'azote par l'hélium $(20 \%$ de $\mathrm{N}_{2}, 80 \%$ de $\mathrm{He}$ ), nous avons pu étendre la gamme d'étude de l'azote jusqu'à $1,4 \mathrm{eV}$.

Un exemple des caractéristiques du générateur à haute enthalpie est donné dans le tableau II, dans le cas d'un faisceau d'hélium, à 300 et à $3000 \mathrm{~K}$.

Ce générateur nécessite des moyens de pompage importants (ils sont indiqués sur la figure 3 ). Ce sont eux qui limitent la densité du gaz sur l'axe du faisceau.

Tableau II. - Caractéristiques du générateur à haute enthalpie.

\begin{tabular}{|c|c|c|}
\hline \multirow{2}{*}{$\frac{\text { Nature du gaz }}{\text { Température du four }}$} & \multicolumn{2}{|c|}{ Hélium } \\
\hline & $300 \mathrm{~K}$ & $3000 \mathrm{~K}$ \\
\hline $\begin{array}{l}\text { Pression du gaz dans le } \\
\text { four }\end{array}$ & 3 bars & 10 bars \\
\hline $\begin{array}{l}\text { Concentration à } 150 \mathrm{~mm} \\
\text { de la tuyère (zone d'inter- } \\
\text { action }\end{array}$ & $2 \times 10^{12} \mathrm{at} / \mathrm{cm}^{3}$ & $3 \times 10^{12} \mathrm{at} / \mathrm{cm}^{3}$ \\
\hline Vitesse hydrodynamique & $1,7 \times 10^{3} \mathrm{~m} / \mathrm{s}$ & $4,8 \times 10^{3} \mathrm{~m} / \mathrm{s}$ \\
\hline $\begin{array}{l}\text { Dispersion des vitesses } \\
\text { (largeur totale à mi- } \\
\text { hauteur) }\end{array}$ & $7 \%$ & $12 \%$ \\
\hline Nombre de Mach & 26 & 15 \\
\hline
\end{tabular}

Pour mesurer la distribution des vitesses des atomes dans la direction du faisceau, nous disposons d'un dispositif de mesure du temps de vol (Figs. 3 et 4). Il se compose d'un hacheur de faisceau et de la sonde d'un spectromètre de masse associés à un analyseur multi- 


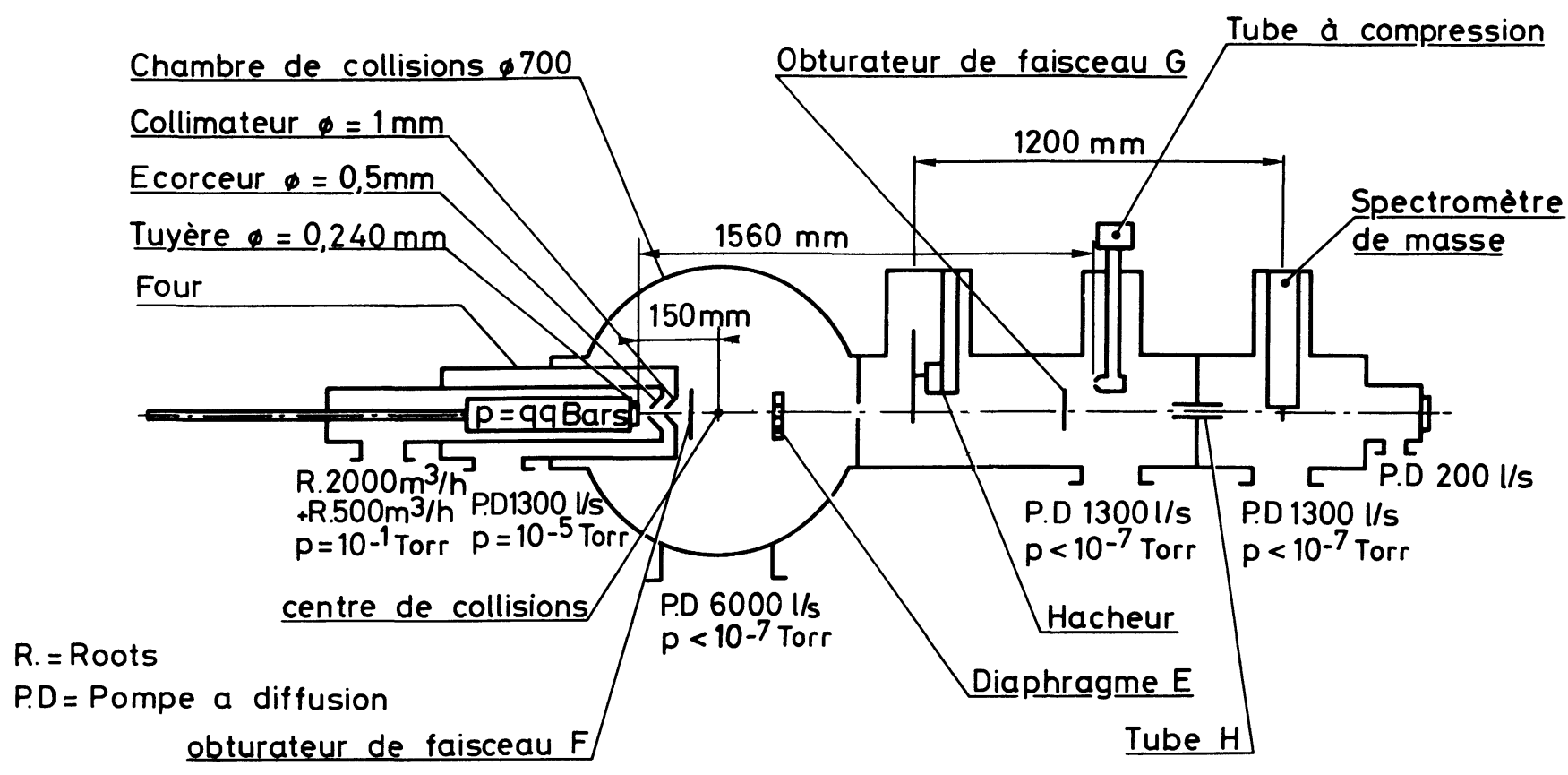

Fig. 3. - Générateur à haute enthalpie et dispositif de mesure du temps de vol et de la densité.

[High enthalpy beam generator and measurement systems for time of flight and density.]

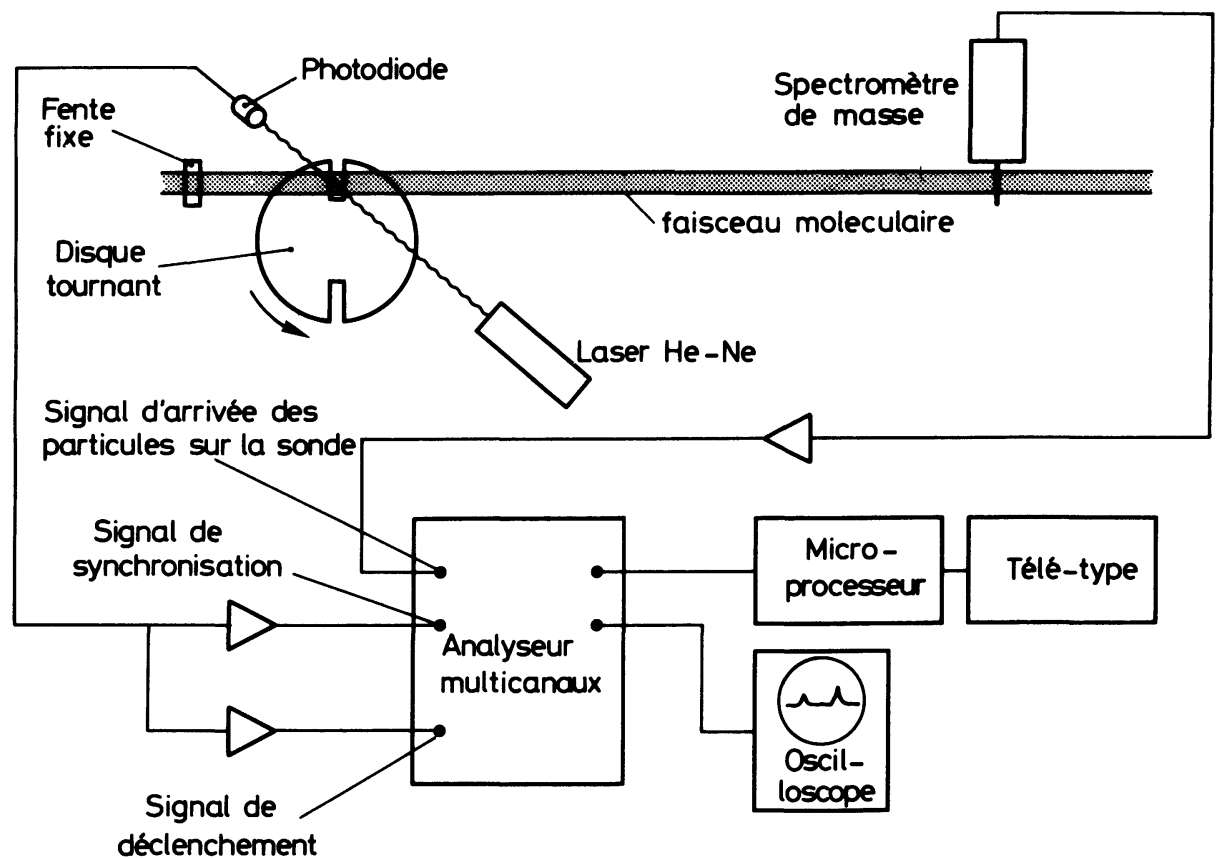

Fig. 4. - Dispositif temps de vol.

[Time of flight apparatus.]

canaux (256 canaux). La synchronisation du déclenchement de l'analyseur sur l'instant du passage de la bouffée de particules au travers des fentes du hacheur est assuré par un petit laser He-Ne. Le signal reçu par la sonde d'un spectromètre de masse peut être visualisé sur un oscilloscope (Fig. 5) sa représentation analytique a été donnée par R. Campargue [5]

$$
S_{\mathrm{QUA}}=\frac{A}{t^{4}} \exp \left(-\frac{m V_{\mathrm{G}}^{2}}{2 K T_{\mathrm{t}}}\right)\left(\frac{t_{0}}{t}-1\right)^{2}
$$

où $T_{\text {t }}$ est la température de translation,

$V_{\mathrm{G}}$ est la vitesse hydrodynamique,

$t$ est le temps de vol,

$t_{0}$ est le temps de vol correspondant à la vitesse hydrodynamique. 


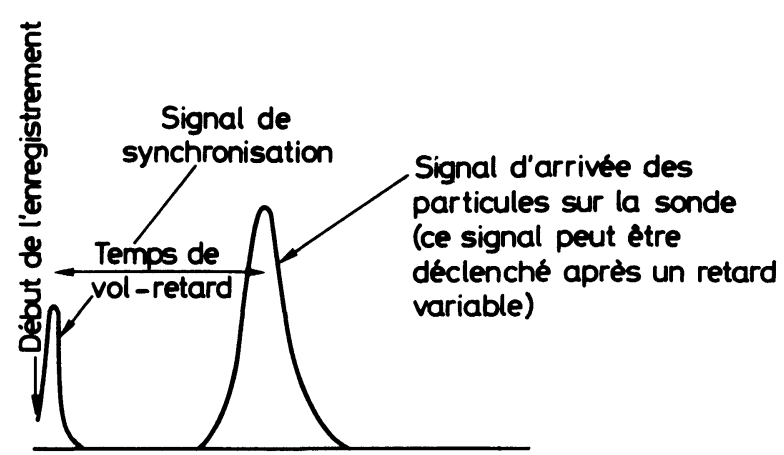

Fig. 5. - Visualisation sur oscilloscope de la courbe de temps de vol.

[Time of flight signal as it is seen on an oscilloscope.]

Les sorties digitalisées de l'analyseur multicanaux permettent un traitement mathématique à partir de la formule (1). Le temps de vol peut ainsi, toujours être connu à moins de $3 \%$.

Pour limiter au maximum les signaux parasites, la sonde du spectromètre de masse doit être pompée très efficacement. Les produits de dégazage ne sont pas à craindre, car ils sont éliminés par la sélection en masse de l'appareil de mesure. Par contre, le gaz apporté par le jet doit être réduit uniquement à ce qui est susceptible de traverser la sonde, c'est pourquoi un diaphragme $\mathrm{E}$ (Fig. 3) limite le faisceau à sa partie axiale. Le hacheur interceptant $99,5 \%$ du faisceau moléculaire, il faut éviter que le gaz ainsi thermalisé puisse parvenir dans l'enceinte de la sonde, c'est pourquoi seul un tube $H$ (Fig. 3) présentant une faible conductance laisse le libre passage au faisceau.

Outre la mesure des temps de vol, le dispositif peut servir à la mesure des flux de particules. En effet à une énergie donnée le signal fourni par la sonde est proportionnel à la densité des particules dans le faisceau [5] pondéré par le coefficient d'efficacité de la collection des ions dans la source d'ionisation. On peut montrer, compte tenu de la faible dispersion des vitesses des particules dans le faisceau que :

$$
Q \propto \frac{N}{t_{0}} A(E)
$$

où $Q$ est le flux des particules,

$N \quad$ est la somme du contenu des mémoires de l'analyseur multicanaux correspondant à l'arrivée des particules sur le détecteur,

$A(E)$ est le coefficient moyen d'efficacité de collection des ions pour l'énergie $E$.

Le coefficient $A(E)$ est une caractéristique de la sonde du spectromètre de masse, il peut être déterminé à l'aide du dispositif du tube de compression décrit ci-après. Il est ainsi possible de déterminer le flux $Q$ à partir des mesures de temps de vol.

L'utilisation d'une sonde de spectromètre de masse comme détecteur dans le dispositif temps de vol $a$, outre l'intérêt d'éliminer les signaux parasites provenant du gaz résiduel, l'avantage de mettre en évidence les différents polymères pouvant être présents dans le faisceau. C'est ainsi que dans un faisceau d'argon il a été possible de mettre en évidence tous les polymères de l'argon $\mathrm{Ar}_{1} \mathrm{à} \mathrm{Ar}_{6}$, la gamme de mesure du spectromètre de masse ne permettant pas d'observer les polymères de l'argon au-delà de l'Ar ${ }_{6}$.

Nous utilisons pour mesurer la densité des atomes ou des molécules de gaz un dispositif à tube de compression. Il se compose d'un conduit de faible conductance ayant $34 \mathrm{~mm}$ de long et $2,5 \mathrm{~mm}$ de diamètre débouchant sur un réservoir de $350 \mathrm{~cm}^{3}$ environ dont la pression est mesurée à l'aide d'une jauge à ionisation. L'axe du conduit étant aligné dans la direction du faisceau, il s'établit un équilibre entre le flux de particules entrant dans le réservoir et le flux de particules en sortant. Le flux entrant est constitué essentiellement par les particules du faisceau et le flux sortant, par ces mêmes particules thermalisées et par le flux de dégazage du réservoir. Ce flux de dégazage peut être apprécié grâce à un obturateur de faisceau G (Fig. 3) placé à l'entrée du tube de compression. Nous désignerons par $\Delta p$ la pression partielle dans le réservoir provenant du faisceau seul, pour bien montrer qu'elle est en fait la différence des pressions dans le réservoir, avec et sans jet.

Cette pression peut s'écrire :

$$
\Delta p=\frac{Q}{C}\left(1-\exp \left[-\frac{C}{V} t\right]\right)
$$

où $Q=n_{\mathrm{G}} V_{\mathrm{G}} S$ est le flux entrant ( $S$ est la section du tube de compression),

$C$ est la conductance du tube de compression,

$V \quad$ est le volume du réservoir,

$t \quad$ est le temps.

Ce dispositif pouvant se déplacer dans un plan perpendiculaire au faisceau, il nous a été possible de tracer les profils de densité du faisceau.

Nous avons pu montrer :

- Que le faisceau existe à l'intérieur d'un cône ayant pour sommet l'orifice de la tuyère et s'appuyant sur le diamètre intérieur du collimateur. La tuyère étant le centre d'homothétie du système et le jet étant libre en aval de l'écorceur, la connaissance du profil à l'entrée du tube de compression permet de déterminer le profil de densité dans la zone d'interaction.

- Que ce profil, aux erreurs expérimentales près, ne dépend dans la zone d'interaction ni de la nature du gaz (Fig. 6) ni de la pression de stagnation, ni de la température du four (Fig. 7).

En régime permanent il y a égalité entre le flux entrant et le flux sortant ce qui donne :

$$
n V_{\mathrm{G}} S=C \cdot \Delta p=Q .
$$

En régime transitoire on voit que la pression d'équi- 


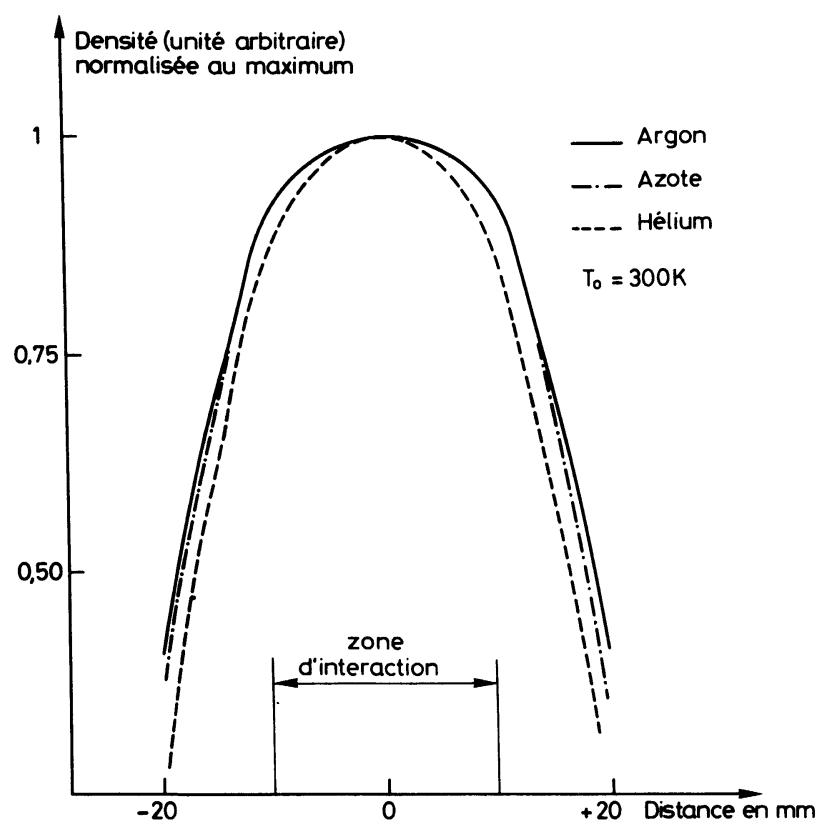

Fig. 6. - Profil de densité dans le faisceau supersonique : Influence de la nature du gaz.

[Density profile of the supersonic beam : Influence of the gas nature.]

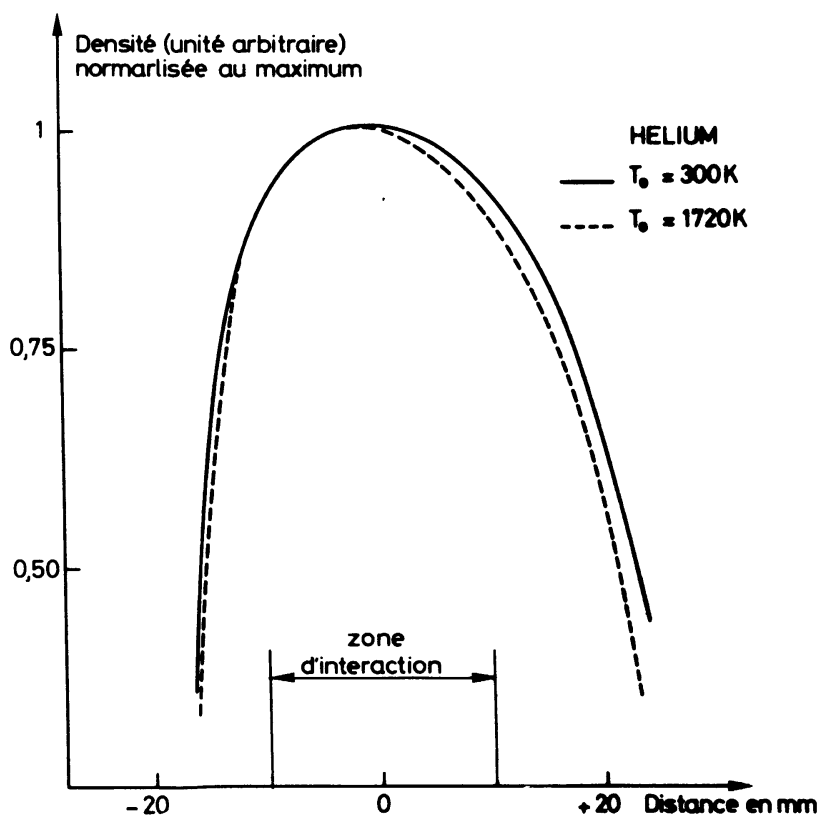

Fig. 7. - Profil de densité dans le faisceau supersonique : Influence de la température du four.

[Density profile of the supersonic beam : Influence of oven temperature.]

libre est d'autant plus rapidement atteinte que la conductance du tube de compression est grande, c'est-à-dire que le gaz est léger. On peut donc déterminer la nature du gaz constituant le faisceau en étudiant la courbe expérimentale $\Delta p=f(t)$. Une vérification a été faite dans le cas de l'azote et de l'hélium. Le rapport des masses moléculaires a été retrouvé à mieux que $5 \%$ près.

L'intérêt de cette remarque se situe au niveau des jets ensemencés. En effet dans le cas d'un mélange de gaz, la composition du mélange dans le four est différente de sa composition dans le jet libre. Si l'on admet que les deux gaz ont un comportement individuel la pression dans le tube à compression devient

$$
p=\sum_{i=\mathrm{He}, \mathrm{N}_{2}} \frac{Q_{i}}{C_{i}}\left(1-\exp \left[-\frac{C_{i} t}{V}\right]\right)
$$

et le courant dans la jauge à ionisation

$$
I=\sum_{i=\mathrm{He}, \mathrm{N}_{2}} Q_{i} \cdot \frac{\sigma(\text { elect. }-i)}{C_{i}}\left(1-\exp \left[-\frac{C_{i} t}{V}\right]\right)
$$

$\sigma($ elect. $-i)$ section efficace electron-gaz correspondant à la tension d'accélération des électrons dans la jauge.

I courant dans la jauge à ionisation.

En comparant les points expérimentaux aux calculs effectués à partir de la formule (6) pour différentes concentrations, il est possible de déterminer par une méthode de moindres carrés la concentration des différents composants du faisceau. C'est ainsi que partant d'un mélange à $20 \%$ d'azote et $80 \%$ d'hélium dans le four, nous avons pu déterminer que la concentration dans le faisceau devenait $50 \%$ d'azote et $50 \%$ d'hélium. D'autre part, nous avons montré que pour ce même mélange la concentration dans le faisceau n'était pas modifiée, ni par la pression, ni par la température du four. De même dans toute la partie utile du faisceau, c'est-à-dire celle qui traverse la zone d'interaction, cette concentration reste constante.

2.3 UNE CHAMBRE DE COLLISION. - Elle est pompée énergiquement par une pompe à diffusion de $6000 \mathrm{l} / \mathrm{s}$ munie d'un piège à azote liquide, la pression du gaz résiduel est ainsi très faible $<5 \times 10^{-8}$ torr. En présence du faisceau de gaz la pression dans cette enceinte est de $10^{-6}$ torr.

2.4 SySTÈME D'EXCITATION LUMINEUSE ET DE COLLECTION DE LA LUMIÈRE DE FLUORESCENCE. - L'excitation des atomes alcalins est réalisée grâce à l'émission lumineuse d'un laser accordable ce qui permet d'obtenir une forte densité d'atomes dans l'état excité désiré, nécessaire pour obtenir une bonne sensibilité de l'expérience de collision projetée.

Les expériences peuvent être réalisées soit avec un laser pulsé (laser à colorant DL $14 \mathrm{P}$ - Molectron + laser à azote UV 12 - Molectron) soit avec un laser continu monomode (laser en anneau 380 SpectraPhysics + laser à krypton ionisé 171 Spectra-Physics).

L'optique d'entrée de la lumière d'excitation est constituée d'un ensemble comprenant deux prismes de Glan et d'une lame 1/4 d'onde. Il est ainsi possible 


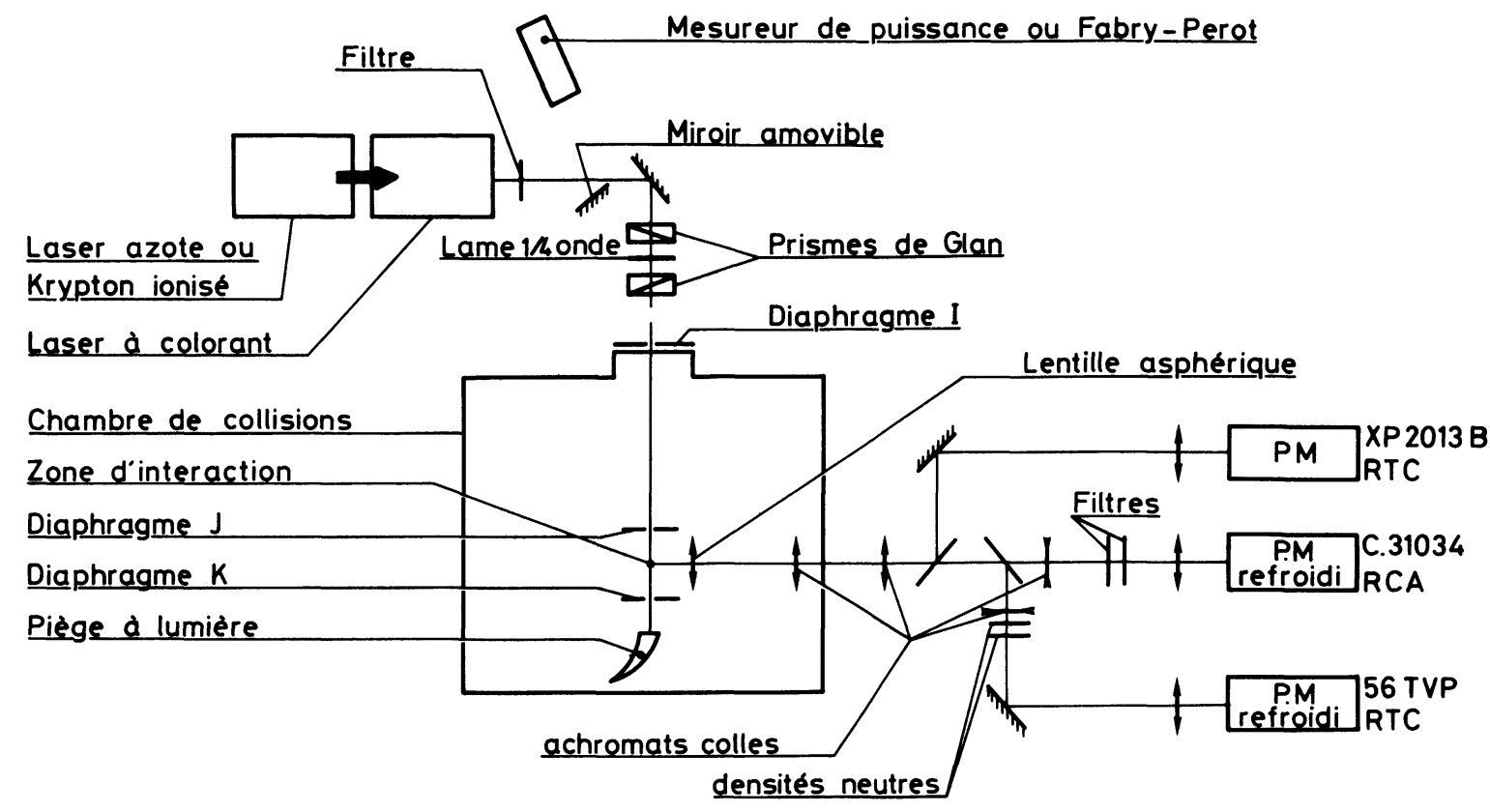

Fig. 8. - Excitation lumineuse et système de détection optique.

[Light excitation and optical detection apparatus.]

de choisir la nature de la lumière excitatrice. De grandes précautions, diaphragmes $\mathrm{I}, \mathrm{J}, \mathrm{K}$, piège à lumière (Fig. 8) doivent être prises pour éviter que la lumière diffusée ne parvienne au détecteur.

Le système de collection de la lumière de fluorescence est représenté sur la figure 8. Deux lames à faces parallèles séparent le faisceau collecté en trois parties. La partie transmise $(85 \%$ du signal) est destinée à la détection du signal de fluorescence sensibilisée. Deux filtres interférentiels (MATRA) isolent le signal de fluorescence directe du signal de fluorescence sensibilisée. Pour profiter au maximum des qualités de sélectivité spectrale de ces deux filtres, il est indispensable que le système optique soit parfaitement réglé et exempt d'aberrations. Une troisième voie de mesure permet, par visualisation sur un oscilloscope, un contrôle permanent du calage en fréquence du laser, sur la raie d'absorption de l'alcalin.

Dans le cas où nous utilisons le laser pulsé, les chaînes de comptage ne sont actives que pendant la durée des signaux de fluorescence. La synchronisation des discriminateurs et du laser est obtenue grâce à un générateur de retard digital mis au point par le LETI. Nous pouvons ainsi obtenir un excellent rapport signal/bruit. Deux obturateurs de faisceaux $D$ et $F$ (Figs. 2 et 3) sont prévus l'un sur le faisceau alcalin, l'autre sur le faisceau de gaz, ils permettent de mesurer l'importance des signaux parasites.

Il est possible pour réaliser des expériences faisant intervenir la polarisation de la lumière excitatrice, de placer la zone d'interaction dans un champ magnétique.

De grandes précautions doivent être apportées aux alignements des différents composants car ils condi- tionnent la réussite de l'expérience. En effet, l'intersection des deux faisceaux de particules et du faisceau laser détermine la zone d'interaction qui doit être centrée sur le dispositif de détection optique. Tout doit donc être parfaitement réglé et énergiquement bloqué car la zone d'interaction est petite $(2 \times 2 \times 3 \mathrm{~mm})$.

3. Exemple d'utilisation de ce dispositif. - Nous nous étions proposé, lors de la conception de réaliser un dispositif expérimental permettant d'étudier dans les meilleures conditions les processus collisionnels atome (alcalin) excité - atome ou molécule.

Nous désirions :

1) Disposer d'un dispositif nous permettant de déterminer les sections efficaces associées à ces processus sur une large gamme d'énergie.

2) Bien connaître les caractéristiques des partenaires de la collision avant que celle-ci se produise.

3) Disposer d'un appareillage nous permettant de mettre en évidence des processus très peu probables : grande sensibilité.

En présentant, rapidement, parmi les résultats que nous avons obtenus $[6,7]$ ceux concernant les couples $\mathrm{Rb}^{*}$-He et $\mathrm{Rb}^{*}-\mathrm{N}_{2}$, nous allons voir que ces objectifs ont été atteints. On s'est proposé de mesurer, la section efficace du transfert de structure fine $5 \mathrm{P}_{1 / 2} \rightarrow 5 \mathrm{P}_{3 / 2}$ se produisant dans l'atome de rubidium excité dans le niveau $5 \mathrm{P}_{1 / 2}$, lors d'une collision avec un atome d'hélium ou une molécule d'azote. Les résultats sont présentés sur la figure 9 .

Nous voyons que le comportement de $\mathrm{He}$ et $\mathrm{N}_{2}$ est extrêmement différent. Dans le cas du couple 


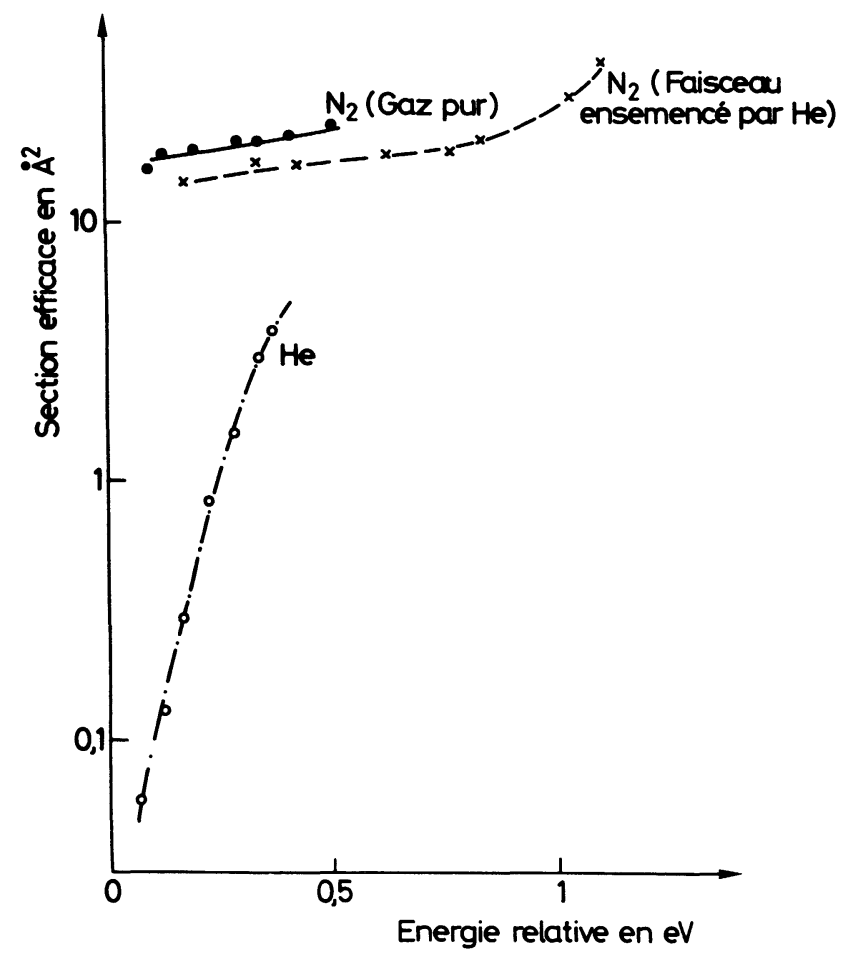

Fig. 9. - Variation des sections efficaces des processus de transition de structure fine $\mathrm{Rb}\left(5 \mathrm{P}_{1 / 2} \rightarrow 5 \mathrm{P}_{3 / 2}\right)$-He et $\mathrm{Rb}\left(5 \mathrm{P}_{1 / 2} \rightarrow 5 \mathrm{P}_{3 / 2}\right)$ $\mathrm{N}_{2}$ en fonction de l'énergie relative.

[Energy dependence of the cross sections for the fine structure transition processes $\mathrm{Rb}\left(5 \mathrm{P}_{1 / 2} \rightarrow 5 \mathrm{P}_{3 / 2}\right)-\mathrm{He}$ and $\mathrm{Rb}\left(5 \mathrm{P}_{1 / 2} \rightarrow 5 \mathrm{P}_{3 / 2}\right)$ $\mathrm{N}_{2}$.]

$\mathrm{Rb}^{*}$-He, nous observons une augmentation très rapide (deux ordres de grandeur) de la section efficace, pour une variation de l'énergie relative de $0,4 \mathrm{eV}$ (processus quasi adiabatique). Cette variation est en excellent accord avec celle déduite par Gallagher [8] d'une expérience en cellule. C'est d'ailleurs l'une de ses valeurs $\left(\sigma=0,3 \AA^{2}\right.$ à $\left.E_{\mathrm{R}}=0,15 \mathrm{eV}\right)$ que nous avons retenue pour calibrer le dispositif. La comparaison de ces résultats expérimentaux avec les calculs faits par Olson [9] a confirmé que les mécanismes intervenant dans les collisions de transfert de structure fine Alcalin excité-gaz rare sont bien pris en compte par la théorie. Les légers écarts subsistant entre les prévisions théoriques et les résultats expérimentaux étant attribués aux faibles incertitudes existant encore dans la connaissance des potentiels d'interaction.
Dans le cas du couple $R b^{*}-N_{2}$ au contraire, la valeur de la section efficace est sensiblement constante quelle que soit l'énergie de la collision et atteint environ $20 \AA^{2}$. Actuellement aucune prévision théorique ne rend compte de façon satisfaisante de ces résultats. Toutefois pour le couple $\mathrm{Rb}^{*}-\mathrm{H}_{2}$ [10] Hickman a justifié les résultats obtenus expérimentalement par la prise en compte d'un nouveau mécanisme. Les premiers résultats obtenus [11] sont en bon accord avec nos résultats expérimentaux et expliquent l'importante différence existant entre les systèmes alcalin excité-gaz rare et alcalin excité-molécule. Nous avons en ensemençant le faisceau d'azote par de l'hélium, étendu la gamme d'énergie étudiée jusqu'à $1,2 \mathrm{eV}$. Le fait que les résultats obtenus à partir d'un faisceau d'azote pur soient différents de ceux obtenus avec un faisceau ensemencé, provient de ce que les molécules d'azote, bien qu'ayant la même énergie cinétique, se trouvent à des températures rotationnelles très différentes dans l'un et l'autre cas.

4. Conclusion. - Nous venons de décrire l'outil dont nous disposons actuellement. S'il est performant, il est néanmoins encore perfectible. Par exemple l'utilisation des possibilités de la spectroscopie laser peut permettre d'étudier de façon précise l'état interne des particules après la collision ou de mesurer des sections efficaces différentielles. Ce dispositif peut également s'adapter, sans modifications trop importantes, à l'étude des collisions réactives.

Ce dispositif n'a pu être construit que grâce au concours du groupe de l'assistance technique et du groupe de l'électronique du service de Physique des atomes et des surfaces. Il doit beaucoup à l'aide aimable qui nous a été apportée par le Dr Campargue et ses collaborateurs. Son financement a été assuré par l'Institut de Recherche Fondamentale du groupe C.E.A. et par le C.N.R.S. dans le cadre de l'A.T.P. « Etats transitoires en physique des collisions » (juin 1974).

Les auteurs de cet article n'ont pas été les seuls à participer à la conception, la réalisation et la mise au point du dispositif expérimental, il faut y associer Mme Benoit, Mlle Perrin, MM. de Mesmay et Grandet, qui nous ont quittés.

Que tous reçoivent ici nos plus sincères remerciements.

\section{Bibliographie}

[1] Pimbert, M., Rocchiccioli, J. L., Cuvellier, J., C. R. Hebd. Sean. Acad. Sci. Paris 270 (1970) B 684.

[2] Pimbert, M., J. Physique 33 (1972) 331.

[3] Cuvellier, J., Fournier, P. R., Gounand, F., Berlande, J., C. R. Hebd. Séan. Acad. Sci. Paris 276 (1973) B 855.

[4] HopPE, H. O., RAPPORT 107 (1973) Max-Planck Institut für Strömungsforschung-Göttingen.

[5] Campargue, R., Thèse Paris (1970).

[6] Cuvellier, J., Berlande, J., Benort, C., Perrin, M. Y.,
Mestdagh, J. M., De Mesmay, J., J. Phys. B. 12 (1979) L 461.

[7] Mestdagh, J. M., Cuvellier, J., Berlande, J., Binet, A., De Pujo, P., J. Phys. B. 13 (1980) 4589.

[8] Gallagher, A., Phys. Rev. 172 (1968) 88.

[9] Olson, R. E., Chem. Phys. Lett. 33 (1975) 250.

[10] Mestdagh, J. M., De Pujo, P., Cuvellier, J., Binet, A. Fournier, P. R., Berlande, J., J. Phys. B. à paraître.

[11] Hickman, A. P., Soumis pour publication à Phys. Rev. Lett. 